\title{
Strong Barrier Coverage of Wireless Sensor Networks
}

\author{
Benyuan Liu ${ }^{\dagger}$ Olivier Dousse* \\ $\dagger$ Department of Computer Science \\ University of Massachusetts Lowell \\ Lowell, MA 01854, USA \\ \{bliu,wang, asaipull\}@cs.uml.edu
}

\author{
Jie Wang ${ }^{\dagger} \quad$ Anwar Saipulla ${ }^{\dagger}$ \\ * Deutsche Telekom Laboratories \\ Ernst-Reuter-Platz 7 \\ 10587 Berlin, Germany \\ olivier.dousse@ieee.org
}

\begin{abstract}
Constructing sensor barriers to detect intruders crossing a randomly-deployed sensor network is an important problem. Early results have shown how to construct sensor barriers to detect intruders moving along restricted crossing paths in rectangular areas. We present a complete solution to this problem for sensors that are distributed according to a Poisson point process. In particular, we present an efficient distributed algorithm to construct sensor barriers on long strip areas of irregular shape without any constraint on crossing paths. Our approach is as follows: We first show that in a rectangular area of width $w$ and length $\ell$ with $w=\Omega(\log \ell)$, if the sensor density reaches a certain value, then there exist, with high probability, multiple disjoint sensor barriers across the entire length of the area such that intruders cannot cross the area undetected. On the other hand, if $w=o(\log \ell)$, then with high probability there is a crossing path not covered by any sensor regardless of the sensor density. We then devise, based on this result, an efficient distributed algorithm to construct multiple disjoint barriers in a large sensor network to cover a long boundary area of an irregular shape. Our algorithm approximates the area by dividing it into horizontal rectangular segments interleaved by vertical thin strips. Each segment and vertical strip independently computes the barriers in its own area. Constructing "horizontal" barriers in each segment connected by "vertical" barriers in neighboring vertical strips, we achieve continuous barrier coverage for the whole region. Our approach significantly reduces delay, communication overhead, and computation costs compared to centralized approaches. Finally, we implement our algorithm and carry out a number of experiments to demonstrate the effectiveness of constructing barrier coverage.
\end{abstract}

\section{Categories and Subject Descriptors}

C.2.1 [Computer-Communication Networks]: Network Architecture and Design-Network topology

Permission to make digital or hard copies of all or part of this work for personal or classroom use is granted without fee provided that copies are not made or distributed for profit or commercial advantage and that copies bear this notice and the full citation on the first page. To copy otherwise, to republish, to post on servers or to redistribute to lists, requires prior specific permission and/or a fee.

MobiHoc'08, May 26-30, 2008, Hong Kong SAR, China.

Copyright 2008 ACM 978-1-60558-083-9/08/05 ...\$5.00.

\section{General Terms}

Performance, Theory

\section{Keywords}

Coverage, sensor networks, algorithm

\section{INTRODUCTION}

Barrier coverage [4] of wireless sensor networks provides sensor barriers guarding boundaries of critical infrastructures or assets, such as country borders, coastal lines, and boundaries of battlefields. In a wireless sensor network, intruders are detected when they enter the areas covered by sensors. The union of the covered areas of sensors forms a barrier. A barrier may contain gaps, allowing the intruder to pass through undetected. A strong barrier has no gaps, so that no intruders can cross the region undetected no matter what crossing paths the intruders would choose. Constructing a strong barrier from sensors randomly deployed in a region is a challenging problem. Whether an intruder can be detected depends on a number of parameters.

First, barrier coverage is affected by sensor deployment methods. In barrier coverage sensors are often deployed in regions of an irregular long belt shape. In certain applications sensors may be manually placed in desired locations and so barrier coverage can be achieved using a minimum number of sensors by aligning them in straight lines crossing the network region. In other applications, sensors may have to be deployed randomly. They may be, for example, dropped by airplanes or launched by artilleries. In random sensor deployments, barrier coverage depends on the spatial distribution of sensor locations.

Second, barrier coverage is affected by the crossing paths taken by the intruder. A crossing path is a path that crosses the complete width of the region from one side to the other side. If an intruder has no knowledge of the sensor locations (i.e., sensors are stealthy), it is proved in [8] that the optimal crossing paths that minimize the probability of being detected in a two-dimensional rectangular network (a.k.a. a strip network) are the orthogonal crossing paths. Recently, Kumar, Lai, and Arora defined two types of barrier coverage [6]. They are weak barrier coverage, which guarantees to detect intruders moving along congruent paths; and strong barrier coverage, which guarantees to detect intruders no matter what crossing paths they take.

Figure 1 illustrates the difference between strong barrier coverage and weak barrier coverage. In the top figure, the network has weak barrier coverage for all orthogonal crossing 
paths (dashed paths). However, there is an uncovered path (solid path) through the region. The bottom figure shows an example of strong barrier coverage where no intruders can cross the region undetected, no matter how they choose their crossing paths. The barrier is highlighted using shaded sensing areas.

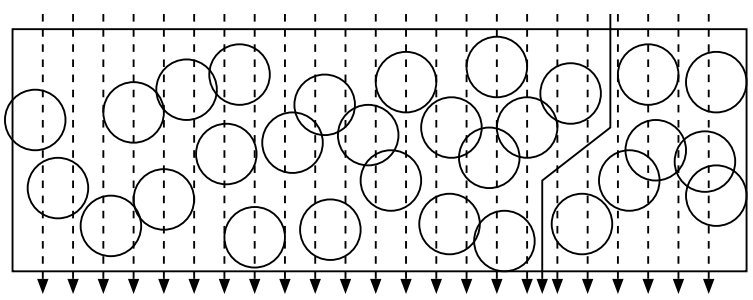

weak coverage

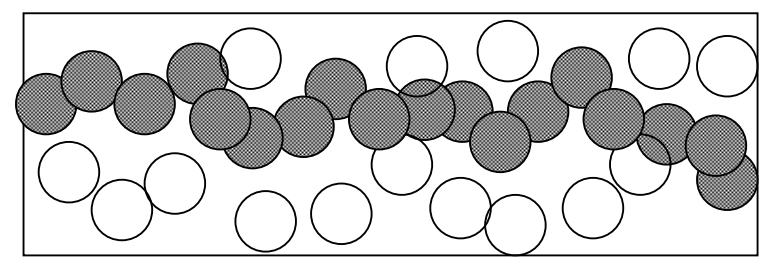

strong coverage

Figure 1: Weak and strong barrier coverage. Weak barrier coverage guarantees to detect intruders on congruent crossing paths. Strong barrier coverage guarantees to detect intruders without any constraint on crossing paths.

Kumar et al. [6] presented a critical condition for weak barrier coverage. But conditions for strong barrier coverage remain an open problem. We solve this problem in this paper. We then use this result to devise a distributed barrier construction algorithm. Our major contributions are described as follows:

First, we derive critical conditions for strong barrier coverage, filling the gap in the understanding of the critical conditions for barrier coverage. For a two-dimensional rectangular region where sensors are placed uniformly and independently at random, we show that the strong barrier coverage of a sensor network depends on its width-to-length ratio. If the width of the rectangular area is asymptotically smaller than the logarithm of the length, then the probability that there exist crossing paths that are not covered by sensors tends to 1 , regardless what sensor density is in the sensor network. That is, the network has no strong barrier coverage. On the other hand, if the width is asymptotically larger than the logarithm of the length, the network has strong barrier coverage when the node density reaches a certain value. There exist multiple disjoint sensor barriers across the entire length of the region such that intruders cannot cross the network undetected. This result provides theoretical foundation to the network performance and planning in connection to barrier coverage. The analytical characterizations of the barrier coverage can be used to determine the number of barriers for a given network deployment. The result can also be used to compute the minimum number of sensors needed to satisfy a given barrier coverage requirement.

Second, we devise an efficient distributed algorithm to construct disjoint barriers in a large sensor network to cover long boundary areas of irregular shapes. Kumar et al [6] demonstrated how to use a centralized algorithm to find disjoint barriers on a open strip. This approach, however, could incur high communication overhead and computation cost on large sensor networks. Chen, Kumar, and Lai [1] recently proposed a localized algorithm to detect all intruders whose movement are confined to a slice of the original strip region. This localized algorithm, however, only provides barrier coverage for slices of bounded length. It does not protect the network against intruders that can move beyond the range of a slice.

Our distributed algorithm provides a complete solution to the barrier construction problem. We divide the original network into a number of small segments interleaved by thin vertical strips. Each segment and vertical strip independently computes the barriers in its own area. We construct horizontal barriers in each segment connected by vertical barriers in neighboring vertical strips. Continuous barrier coverage for the whole region is thus guaranteed. By dividing the network into small segments and letting each segment conduct communication and computation independently, our algorithm can significantly reduce delay, communication overhead, and computation costs compared to the early centralized approach. Also, the vertical barriers between neighboring segments improve the robustness of the barrier coverage as they prevent intruders from moving to adjacent segments. Moreover, since each segment computes the barriers between vertical barriers at the two ends, a larger number of local barriers will be obtained, resulting in strengthened local barrier coverage for each segment.

The rest of the paper is organized as follows. In Section 2 we review previous work on the barrier coverage of sensor networks. In Section 3 we describe the network model and define barrier coverage. In Section 4 we derive the critical conditions for strong barrier coverage. In Section 5 we present an efficient algorithm to construct strong barriers. The performance evaluation is presented in Section 6. Finally, Section 7 concludes the paper.

\section{RELATED WORK}

In this section we review the previous research on the barrier coverage of wireless sensor networks. The notion of barrier coverage was first introduced in the context of robotics sensors [4]. The goal of barrier coverage is to detect intruders that attempt to cross from one side of a region to the opposite side. Several different barrier coverage measures and the related issues have been studied.

In [9], path coverage is defined. Efficient algorithms are proposed to find maximum breach or support paths between two end points that are least or most likely to be detected by sensors. In [10], the notion of path exposure is introduced to measure the likelihood that an intruder is detected when moving along a given path. A centralized algorithm is proposed to find the minimum exposure paths, where the probability of an intruder being detected is minimized. These path coverage problems are further studied in [7, 12], where efficient distributed algorithms are proposed.

In [8], Liu and Towsley first studied the barrier coverage of two-dimensional plane and two-dimensional strip sensor networks using percolation theory results. The barrier coverage of a two-dimensional plane network is related to the existence of a giant sensor cluster that percolates the network. However, the strength of the barrier coverage, i.e., the 
number of disjoint barriers, was not obtained. For a twodimensional strip network of finite width, it is proved that there always exist crossing paths along which an intruder can cross the strip undetected. Furthermore, the probability that an intruder is detected when crossing a strip is characterized.

The most related work to ours is by Kumar et al. [6] and Chen et al. [1]. Kumar et al. devised a centralized algorithm to determine whether a region is $k$-barrier covered, and derived the critical conditions for weak barrier coverage in a randomly deployment sensor network. But the centralized algorithm could incur high communication overhead and computation cost on large sensor networks, and conditions for strong barrier coverage remain an open problem. Recently, Chen et al. devised a localized algorithm that guarantees the detection of intruders whose trajectory is confined to a slice of the belt region of deployment. It does not protect the network against intruders that can move beyond the range of the thin slices.

\section{NETWORK MODEL}

We assume that sensors are deployed in a two-dimensional strip area of size $A_{2 \text {-dim strip }}=[0, n] \times[0, w(n)]$. A twodimensional rectangular area is also referred to a strip. We can adjust the width $w(n)$ to obtain different width to length ratios. More realistic network scenarios may be approximated by a combination of different strip shapes. We consider the static sensor network scenario where sensors do not move after the initial deployment. We assume that sensor nodes are randomly distributed according to a Poisson point process of density $\lambda$. Thus, the expected number of nodes in the network is $\lambda n w(n)$. We use asymptotic analysis when $n$ goes to infinity.

We use the widely adopted Boolean sensing model. Under this model, each sensor is assumed to have a certain sensing range, $r$. A sensor can only sense the environment and detect intruders within its sensing area. A location is said to be covered by a sensor if it lies within the sensor's sensing area. The space is partitioned into two regions, the covered region, which is the region covered by at least one sensor, and the vacant region, which is the complement of the covered region.

We say that two sensors at locations $X_{i}$ and $X_{j}$ are connected if the sensing areas of the two sensors overlap, or equivalently, if $\left|X_{i}-X_{j}\right| \leq 2 r$, where $\left|X_{i}-X_{j}\right|$ is the distance between the two sensors. A sensor barrier is defined to be a connected component of sensors that intersect both of the left and right boundaries of the strip, as depicted in Figure 1. An intruder cannot go through a sensor barrier without being detected, since it will need to go through the sensing area of sensors and thus be detected.

A crossing path is a path that connects one side of the region to the opposite side, where the ingress point and the egress point reside on two opposite sides of the region. For a two-dimensional strip, we assume that the intruders attempt to cross the width of the strip.

The strength of the barrier coverage of a sensor network can be measured by the number of times that an intruder is detected when traversing along a crossing path. A path is said to be $k$-covered if it intercepts at least $k$ distinct sensors. We say an event occurs with high probability (w.h.p.) if its probability tends to 1 as $n \rightarrow \infty$.
Definition 1. A sensor network is said to be strongly $k$-barrier covered if

$P($ any crossing path is $k$-covered $)=1 w . h . p$.

\section{CRITICAL CONDITIONS FOR STRONG BARRIER COVERAGE}

In this section, we present and prove our results on the critical conditions for strong barrier coverage.

THEOREM 1. Consider a sensor network deployed on a two-dimensional rectangular area $A_{2 \text {-dim strip }}=[0, n] \times$ $[0, w(n)]$, where sensors are distributed according to a Poisson point process with density $\lambda$.

- If $w(n)=\Omega(\log n)$, the network is strongly barrier covered w.h.p. when the sensor density reaches a certain value. There exists a positive constant $\beta$ such that w.h.p. there exist $\beta w(n)$ disjoint horizontal sensor barriers crossing the strip.

- If $w(n)=o(\log n)$, the network has no strong barrier coverage w.h.p. regardless what the sensor density is in the underlying sensor network. That is, w.h.p. there exist crossing paths that an intruder can cross the strip without being detected.

It follows from Theorem 1 that the existence and strength of the strong barrier coverage depends on the width-to-length ratio of the strip region. The critical condition for strong barrier coverage to exist is when the width of the strip becomes asymptotically larger than the logarithm of the length, i.e., $w(n)=\Omega(\log n)$. The number of horizontally connected sensor clusters (barriers) is proportional to the width of the strip by a constant factor. On the other hand, when the width is asymptotically smaller than the logarithm of the width, there is no strong barrier coverage, i.e., there exist crossing paths that an intruder can cross the strip without being detected.

If $w(n)=\Omega(\log n)$, the network area can be divided into horizontal rectangles $R_{n}$ of size $n \times \kappa r \log \frac{n}{r}$, for some $\kappa>0$. We will see later from the proof of Theorem 1 that there exist $\beta \kappa \log \frac{n}{r}$ disjoint barriers in each rectangle and hence $\beta w(n) / r$ disjoint barriers in the whole strip, where $\beta=1-$ $\frac{2(\kappa \log 6+2)}{\lambda r^{2}}$. This result can be used to answer a number of sensor deployment questions:

- How many barriers are present in the underlying sensor network?

- How does the number of barriers grow if more sensors can be added?

- What is the minimum number of sensors needed to achieve a given strong barrier coverage?

Proof of Theorem 1. We first convert the barrier coverage problem to a bond percolation model and use the results presented in [3] to complete the proof. Barrier coverage of a strip sensor network is directly related to the number of disjoint connected sensor clusters that cross the width of the strip horizontally. Two sensors are connected if their sensing areas overlap. Each of such sensor clusters acts as "trip wire" that can detect any crossing intruders. As in [3], 

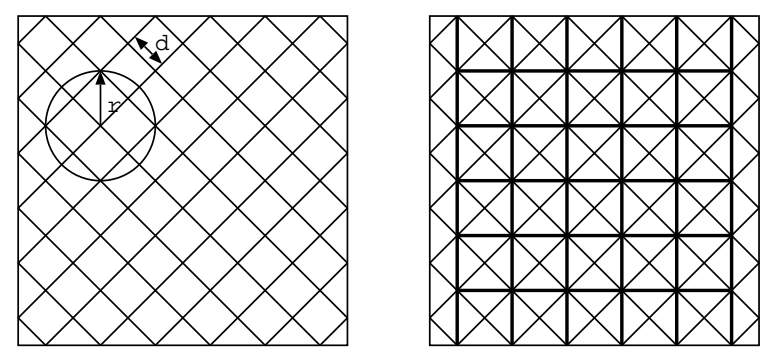

Figure 2: Construction of the bond percolation model.

we construct a bond percolation model to obtain the number of disjoint sensor clusters crossing the length of the strip.

We divide the area into squares of equal size, where the length of each side $d=r / \sqrt{2}$, as depicted in Figure 2. By adjusting sensor density $\lambda$, we can adjust the probability that a square contains at least one sensor:

$$
\begin{aligned}
p & =P(\text { a square contains at least one sensor }) \\
& =1-e^{-\lambda d^{2}}=1-e^{-c^{2}},
\end{aligned}
$$

where $c^{2}=\lambda d^{2}=\lambda r^{2} / 2$.

A square is said to be open if it is occupied by at least one sensor, and closed otherwise. Since side length of each square is $r / \sqrt{2}$, the whole square will be covered by a sensor if it is open. Obviously, if two adjacent squares are both occupied by sensors, the sensing areas of the sensor would overlap and no intruder can cross between the two squares without being detected.

The above construction can now be mapped to a discrete bond percolation model as follows. Horizontal edges are added across half of the squares while vertical edges are added across others, as shown in the right-hand-side of Figure 2. This construction results in a grid of horizontal and vertical edges. A path consists of a sequence of consecutive edges. A path is said to be open or closed if it contains only open or closed edges respectively. Since the squares along an open path are all completely covered by sensor, a crossing open path from left to right of the strip acts as a barrier (or trip wire) that can detect any crossing intruders. The strength of the strong barrier coverage of a strip sensor network thus depends on the number of disjoint open paths.

$\kappa \log m$
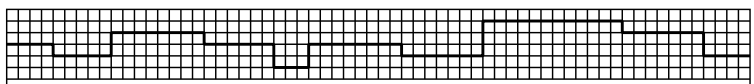

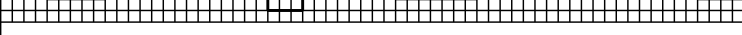

:

Figure 3: The strip network is divided into $\frac{w(n)}{\kappa r \log \frac{n}{r}}$ horizontal rectangles of lattice size $m \times \kappa \log m$ where $m=\frac{n}{r}$. A left to right crossing of Rectangle $R_{n}$ is shown.

If $w(n)=\Omega(\log n)$, we can divide the network area into horizontal rectangles $R_{n}$ of size $n \times \kappa r \log \frac{n}{r}$, for some $\kappa>0$.
There are $\frac{w(n)}{\kappa r \log \frac{n}{r}}$ such rectangles. Let $m=\frac{n}{r}$, each rectangle $R_{n}$ is of lattice size $m \times \kappa \log m$ in the bond percolation model, as illustrated in Figure 3. The following lemma gives the number of disjoint open paths that cross each rectangle. The proof is similar to the proof of Theorem 3 in [3], which will be omitted here.

Lemma 1. For any $\kappa>0$, if $\lambda>2(\log 6+2 / \kappa) / r^{2}$, there exists a strictly positive constant $\beta(c, \kappa)$ such that w.h.p. there exist $\beta \kappa \log m=\beta \kappa \log \frac{n}{r}$ disjoint sensor clusters that cross each Rectangle $R_{n}$ from left to right.

For each rectangle $R_{n}$ of width $\kappa r \log \frac{n}{r}$, there exist $\beta \kappa \log \frac{n}{r}$ disjoint sensor clusters that cross the rectangle. So the total number of such disjoint sensor clusters is $\beta w(n) / r$, which is linearly proportional to the width of the strip.

If $w(n)=o(\log n)$, a simple adaptation of Theorem 11.55 in [5], p. 304, establishes that the probability that there is a path connecting the left and right sides of the strip is zero as $n \rightarrow \infty$, and therefore excludes the existence of the sensor clusters that cross the strip. There exist crossing paths that do not intercept any sensor such that intruders can cross the strip without being detected.

Our strong barrier coverage results are based on an extended network model where node density is kept constant while the network size increases to infinity. In the previous study of critical condition for weak barrier coverage [6], the results are based on a network model of dimension $s \times(1 / s)$ where the area of the network is kept constant while $s \rightarrow \infty$. To better link our results to those of [6], our network model can be rescaled to yield the critical conditions for strong barrier coverage of the $s \times 1 / s$ network model. The result is presented in the following corollary.

COROLlary 1. Consider a two-dimensional strip sensor network of size $s \times 1 / s$ where sensors are randomly distributed according to a Poisson point process of density $\lambda$ and each sensor covers a disk of radius $r$. There exists $\theta>0$, if $\lambda=\theta s^{2} \log ^{2} s$ and the radius $r=1 /(s \log s)$, the strip is strongly barrier covered as $s \rightarrow \infty$. The number of barriers is of order $\log s$.

Proof. Based on Lemma 1, denoting $m=n / r$, if $\lambda>$ $2(\log 6+2 / \kappa) / r^{2}$ for some $\kappa>0$, there exist a total number of $\beta \kappa \log m$ barriers in a rectangle region $R_{n}$ of size $n \times$ $\kappa r \log m$.

To establish the strong barrier coverage result in the network model $R_{s}$ of size $s \times 1 / s$, we let the length to width ratios of the rectangle $R_{n}$ and $R_{s}$ to be asymptotically the same, i.e., $n / \log n=s^{2}$. Therefore, the two network models have the same length to width radio by transformation $s=\sqrt{n / \log n}$, or conversely, $n=s^{2} \log s$.

Now we need to rescale the sensor density and sensing radius in $R_{n}$ to $R_{s}$. In $R_{n}$, the rectangle has length $n$ and constant sensor density and radius. In $R_{s}$, the length is $s=\sqrt{n / \log n}$. Both the length and width of rectangle $R_{n}$ are scaled by a factor of $s / n=s /\left(s^{2} \log s\right)=1 /(s \log s)$ in $R_{s}$. Therefore, the sensor density should be scaled by a factor of $s^{2} \log ^{2} s$ and the sensing radius should be rescaled by a factor of $1 /(s \log s)$. The number of barriers is on the order of $\log s$. 


\section{CONSTRUCTING BARRIERS}

Typical wireless sensors are powered by conventional batteries, and thus they are energy stringent. It is therefore important to schedule sensors so that at any given moment there are just enough active sensors to cover the barrier. Other sensors will be set to the sleep mode to save energy for future use. This way, we can prolong the operation lifetime of the network. Different from the sensor scheduling for area coverage and weak barrier coverage, scheduling sensors for strong barrier coverage requires that sensors used on the same barrier be synchronized to wake up or to sleep simultaneously. Otherwise the barrier will contain holes, which defeats the objective of the strong barrier coverage. Hence, it is important to find sets of sensors so that each of which forms a disjoint barrier. These sets of sensors can then take turn to form a barrier. Moreover, we want to find a scheduling to provide strong barrier coverage with low communication overhead and computation cost.

In [6], the authors show that whether a sensor network is strongly $k$-barrier covered cannot be determined using local algorithms. They convert the $k$-barrier coverage testing problem to the $k$-connectivity testing problem and refer to [11] for the best known global algorithms, which incur a time complexity of $O\left(k^{2}|V|\right)$ for a graph of $|V|$ nodes. To use the algorithm, each sensor node must broadcast its neighbor information to the whole network to construct the connectivity graph of the network. For a connected graph $G(V, E)$ of a sensor network, the message complexity (communication overhead) is $O(|V||E|)$ if the location of each node is broadcast to all of the other nodes, and the end-to-end delay of each message is proportional to the length of the strip. The communication overhead and delay can be formidably high for a large sensor network.

To reduce delay, communication overhead, and computation costs for finding disjoint barriers in a large sensor network, we cover the region to be protected by strips and divides each strip into small segments interleaved by thin vertical strips, as illustrated in Figure 5. Each segment and each vertical strip independently computes the barriers, where a segment computes horizontal barriers and a vertical strip computes both "horizontal" and "vertical barrier" (of course these barriers do not have to be on straight lines). Horizontal barriers in strip segments are connected by vertical barriers in vertical strips to provide continuous barrier c overage across the whole network.

We first present an algorithm to find all disjoint barriers in a strip segment. Each node broadcasts its location and sensing range to all the sensor nodes in the segment. Alternately, each segment can select a node as the delegate for the entire segment and each node sends its location and sensing range to the delegate node. The location of a sensor node can be obtained by on-board GPS device or computed using a node localization scheme. After receiving the location information from other nodes in the segment, each node (or the delegate node) constructs a flow network $G(V \cup\{s, d\}, E)$ by making each sensor node a vertex in $V$ with a vertex capacity of 1 . For any two vertices $u$ and $v$ in $V$, if their sensing areas overlap, connect them with an edge capacity of 1 . We add two nodes $s$ and $d$. For each node $u \in V$, if its sensing area intersects with the left boundary of the segment, connect $s$ to $u$ with an edge capacity of 1 ; if its sensing area intersects with the right boundary of the segment, connect $u$ to $d$ with an edge capacity of 1 . Figure 4 illustrates the construction of a flow network. The vertex capacity is used to ensure that each sensor node can only be used at most once in a barrier when finding a maximum flow from $s$ to $d$. This flow network can be transformed to a traditional flow network by replacing each node $u \in V$ with two nodes $u^{\prime}, u^{\prime \prime}$ and between them a new edge $\left(u^{\prime}, u^{\prime \prime}\right)$ of capacity 1 , where $u^{\prime}$ has all the incoming edges of $u$, and $u^{\prime \prime}$ has all the outgoing edges of $u$. We then use a standard algorithm (e.g., Edmond-Karp or the relabel-to-front algorithms [2]) to find a maximum flow from $s$ to $d$ and all the paths used in the maximum flow. Based on the above construction, nodes of the same path form a barrier that connects the left and right boundaries of the segment, and the maximum flow is the number of available disjoint barriers.

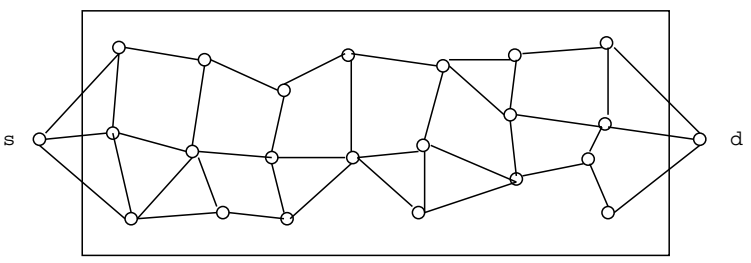

Figure 4: Construction of flow networks. Sensors whose sensing areas intersect with the left and right boundaries are connected to $s$ and $t$, respectively. Each edge and vertex is of capacity 1 . The maximum flow from $s$ to $d$ gives the number of disjoint barriers. Sensors on the same flow path form a barrier.

The complexity of the relabel-to-front algorithm is $O\left(|V|^{3}\right)$. Since the number of sensor nodes deployed in a strip segment is much smaller than the number of sensor nodes deployed in the entire sensor field, this computational complexity would be much more manageable by sensor nodes. This algorithm can be easily modified to find all vertical barriers in a vertical strip by connecting $s$ to all sensors whose sensing areas intersect with the top boundary and connecting $d$ to all sensors whose sensing areas intersect with the bottom boundary. We refer this algorithm as ComputeBarriers in the rest of the paper.

We now describe our divide-and-conquer approach to constructing disjoint barriers in a large strip sensor network.

Divide-and-Conquer Algorithm to Construct Barrier Coverage

1. Divide the given (curly) strip into small segments interleaved by thin vertical strips. The length of each vertical strip is $w(n)$, the width of the original strip. The width of each vertical strip is chosen to be of the order $\log w(n)$ such that there exist $\Theta(\log w(n))$ disjoint barriers crossing the vertical strip according to Theorem 1.

2. In each vertical strip, sensor nodes use ComputeBarriers to find all of the disjoint vertical barriers and the horizontal barriers that connect the vertical barriers together. This computation is carried out in each vertical strip independently.

3. For each strip segment, use ComputeBarriers to find disjoint horizontal barriers intersecting the vertical barriers on both ends of the segment. This computation is carried out in each strip segment independently in parallel. 


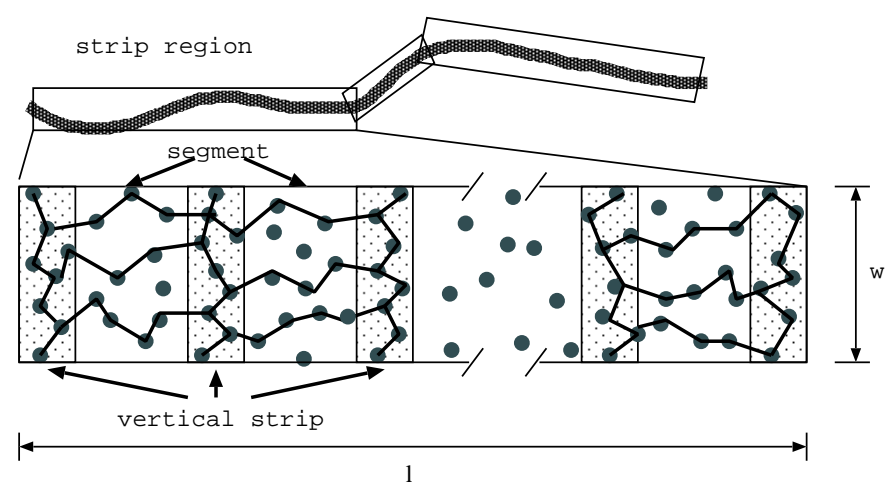

Figure 5: The original strip is divided into small segments interleaved by thin vertical strips. Each vertical strip finds its horizontal and vertical barriers. Each segment finds the local horizontal barriers intersecting the vertical barriers on both ends. These local horizontal barriers are connected by vertical barriers so continuous barrier coverage across the whole strip is ensured. Each dot represents the location of a sensor. For conciseness, sensing areas of sensors are not shown in this figure.

In the above barrier construction process, each segment and vertical strip independently computes the horizontal barriers. These horizontal barriers are connected by vertical barriers in the neighboring vertical strips to provide global barrier coverage. This ensures that there is no gap between the horizontal barriers; so continuous barrier coverage across the whole strip is provided.

The ComputeBarriers algorithm finds all barriers in each strip segment and each vertical strip. If only $k$ disjoint barriers are required, we can activate $k$ horizontal barriers in each segment and in each vertical strip and rotate the active-duty barriers among all available barriers. Also, we can move the vertical strips as sliding windows to avoid the overuse of the same vertical barriers. The barrier rotation process and sliding vertical barrier scheme will balance the power consumption among sensors and hence extend the network lifetime.

Compared to the centralized approach that computes barriers for the whole strip, the above divide-and-conquer approach has the following advantages:

- Lower communication overhead and computation costs. By dividing the large network area into small segments, the message delay, communication overhead, and computation cost can be significantly reduced. The location and sensing area information of a sensor node only need to be broadcast within the strip segment (or within the thin vertical strip) where the node is located, resulting in a smaller delay and communication overhead compared to the whole network broadcasting. For a connected graph $\mathrm{G}(\mathrm{V}, \mathrm{E})$, the communication overhead (for location information broadcast) and computation complexity of the ComputeBarriers algorithm is $O(|V||E|)$, and $O\left(|V|^{3}\right)$, respectively. If the original strip is divided into $n_{s}$ segments interleaved by thin vertical strips, each segment contains less than $|V| / n_{s}$ nodes and $O\left(|V| / n_{s}\right)$ links. The communication overhead is $O\left(|V|^{2} / n_{s}^{2}\right)$, a $n_{s}^{2}$ factor reduc-
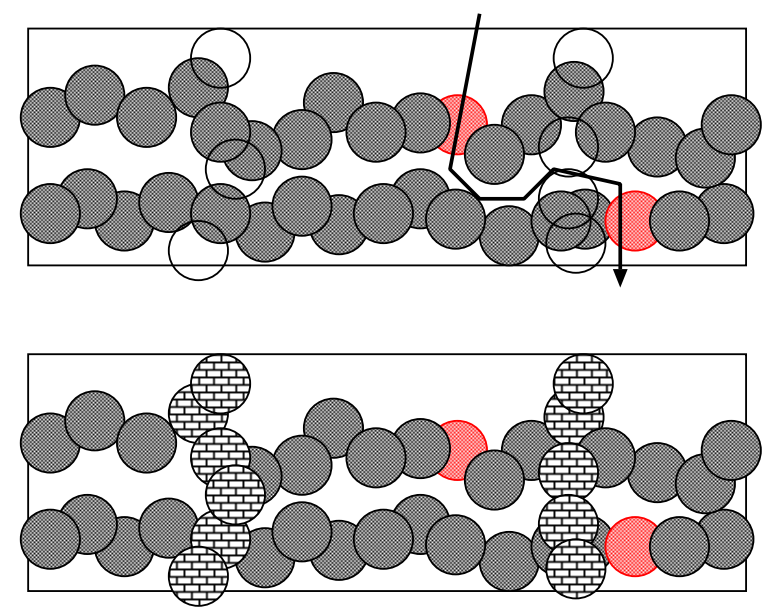

Figure 6: Improved robustness for barrier coverage. Vertical barriers serve as "firewalls" that prevent intruders from moving from one segment to adjacent segments, resulting in improved robustness in case of barrier failures. Shaded nodes indicate activated sensors. Red (lightly shaded) nodes indicate failed sensors. Non-shaded nodes indicate non-activated sensors.

tion from the centralized approach. The computation complexity is $O\left(|V|^{3} / n_{s}^{3}\right)$, a $n_{s}^{3}$ factor reduction from the centralized approach.

- Improved robustness of the barrier coverage. In a centralized approach which constructs global horizontal barriers for the whole strip, a horizontal sensor barrier could be broken if some nodes on the barrier fail, or become compromised or displaced by adversaries. In our divide-and-conquer approach, the original strip is divided into segments by interleaving vertical barriers. In case of node failure, these vertical barriers act as "firewalls" that prevent intruders from moving from its current segment to adjacent segments. This limits the barrier damages within the local segment and hence improving the robustness of the barrier coverage. A scenario of improved robustness with vertical barriers is illustrated in Figure 6.

- Strengthened local barrier coverage. By dividing the original strip into small segments and computing barriers in each segment, a larger number of local horizontal barriers will be found in each segment than for the whole strip. These local barriers are not necessarily part of the global barriers for the whole strip, whose number remains unchanged. Since adjacent segments are blocked by interleaving vertical barriers, a larger number of local barriers results in a strengthened local barrier coverage for each segment. The simulation results in Section 6 confirms that there is significant improvement of local barrier coverage in each segment over global barrier coverage.

\section{PERFORMANCE EVALUATION}

In this section, we first present simulation results on the conditions of strong barrier coverage. We then investigate 
the improvement of local barrier strength in each segment over the global barrier strength. These results will help network planners get a sense of the critical condition of strong barrier coverage, the strength of the barrier coverage as a function of various network parameters, as well as the benefit of our divide-and-conquer approach to constructing barrier coverage.

In the simulation, sensor nodes are distributed into the network of size $l \times w$ according to a two-dimensional Poisson point process of density $\lambda$. The mean number of nodes is $m=\lambda l w$. Each sensor has a sensing range of $r$. By varying the network parameters, $(\lambda, l, w, r)$, we can obtain a wide range of network scenarios. In each simulation, ComputeBarriers algorithm is used to find the number of disjoint barriers in the network. For every network scenario $(\lambda, l, w, r)$, the simulation is repeated 500 times to compute the mean values. The corresponding standard deviations are relatively small and not plotted.

\subsection{Conditions for strong barrier coverage}

In the experiments, we set the length of the region $l=$ 10,000 meters and the sensor's sensing range $r=10$ meters. The width of the strip is varied region from 50 meters to 1000 meters. This is repeated for several different node densities $\lambda=0.005,0.0075$, and 0.01 .

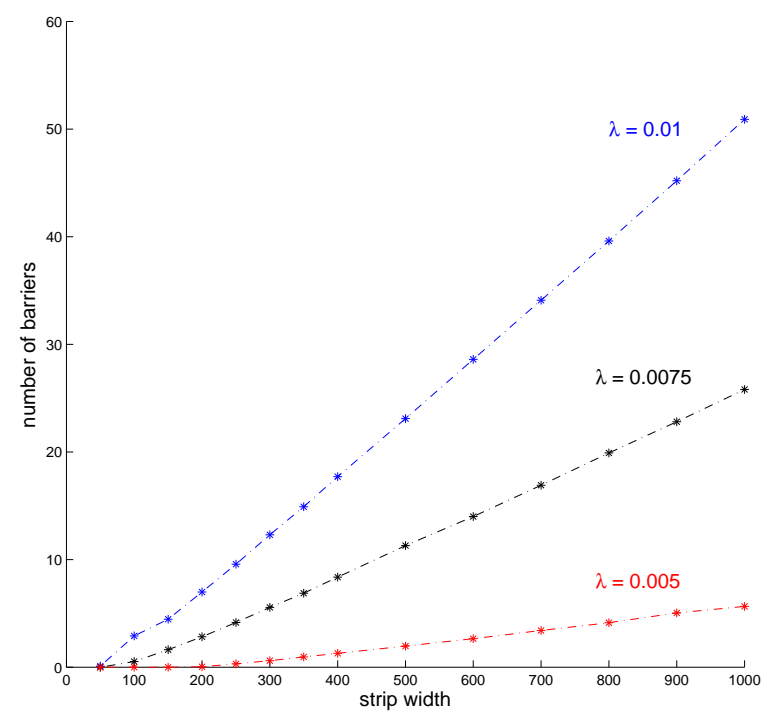

Figure 7: Conditions for strong barrier coverage. Horizontal barriers start to appear only when the width is large enough. The number of barriers increase linearly with the width. For a given width, the network is strongly barrier covered when the density is large enough.

Figure 7 shows the number of disjoint horizontal barriers as a function of the strip width. It can be observed that for each of the node densities, there exists a critical width. The horizontal barriers only start to emerge when the strip width is larger than the critical value. The larger the node density, the smaller the critical width beyond which horizontal barriers start to appear. As the width increases, the number of disjoint horizontal barriers at each node density increases linearly. Also, it can also be observed that for a given width, the network is strongly barrier covered only when the density is large enough. These observations are consistent with the results in Theorem 1.

\subsection{Strengthened local barrier coverage}

In our divide-and-conquer barrier construction algorithm, the whole strip region is divided into small segments interleaved by thin vertical strips. We first compute the vertical barriers in the vertical strips and then compute the horizontal barriers in each segment connected by the vertical barriers in the neighboring vertical strips. Compared to the original strip region, each segment has a larger width-tolength ratio with the same node density. From results in Section 6.1, a larger number of local barriers is expected for each segment than the global barriers for the original strip. This will result in an strengthened barrier coverage for each segment.

In the simulation, we consider network scenarios of size 10,000 meters $\times 250$ meters and node density $\lambda=0.005$, 0.0075 , and 0.01 . The original strip is divided into ten segments interleaved by vertical strips. The length of each vertical strip is varied from 20 meters to 350 meters. The length of each segment is set accordingly. We measure the improvement of barrier strength in each segment over the centralized approach by the barrier improvement ratio, defined to be the number of horizontal barriers in each segment divided by the number of global barriers for the whole strip.

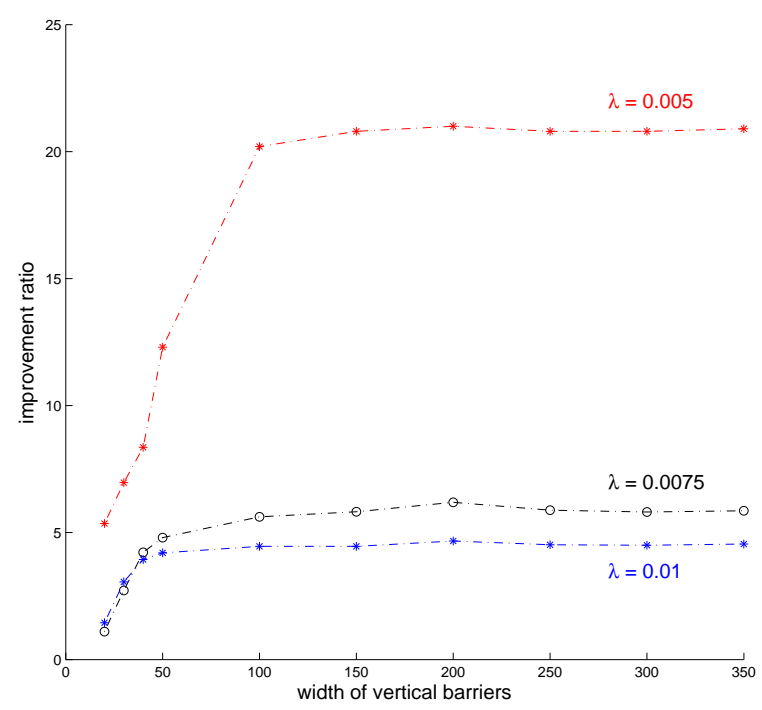

Figure 8: Barrier improvement ratio. Each segment has more local barriers than the global barriers for the whole strip region. These local barriers in each segment are connected by neighboring vertical barriers to provide global barrier coverage.

Figure 8 shows how barrier improvement ratio changes with the width of the vertical strip. It can be observed that the number of barriers is immediately increased as soon as the vertical strips are activated in our divide-and-conquer approach. The barrier improvement ratio continues to in- 
crease as the width of the thin vertical strip increases, and quickly levels off after some point. This is because as the width of the vertical strips increases, there will be more vertical barriers in each vertical strip, and thus a larger number of local barriers in each segment will be connected by these vertical barriers. However, after a certain point, most of the local barriers are already connected by vertical barriers. As a result, the barrier improvement ratio levels off. For node density $\lambda=0.01$, the average number of global barriers for the whole strip in the centralized approach is 9.6. In our divide-and-conquer approach, with a vertical strip width of 100 meters, the average number of horizontal barriers in each segment reaches 42.7 , a more than four-fold increase over the centralized approach. The barrier improvement ratio is even more significant for smaller node densities, for example, the improvement ratio is close to 20 for node density $\lambda=0.005$. This is because there are fewer global barriers for smaller node densities, allowing more room for improvement in each segment.

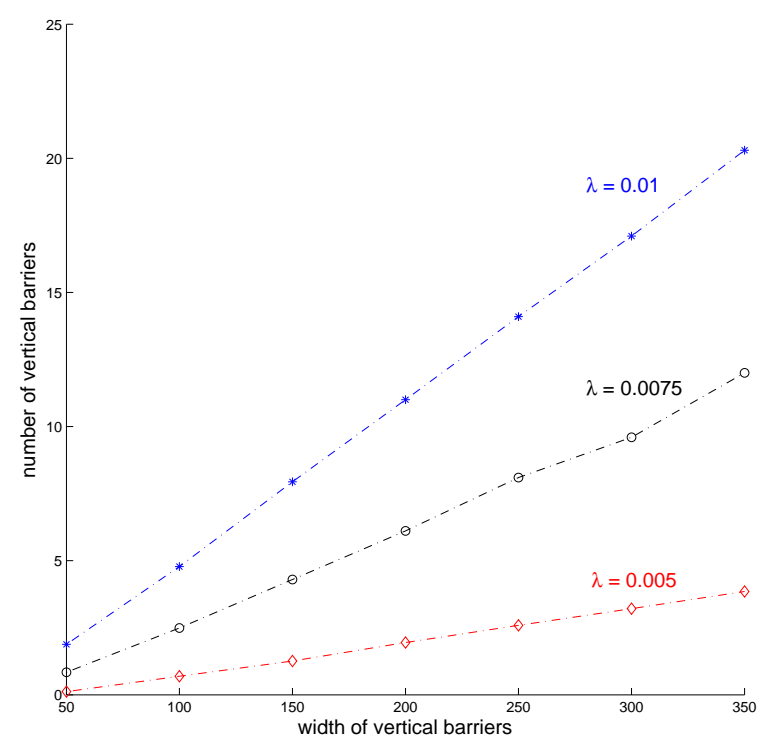

Figure 9: Number of vertical barriers in each vertical strip.

Compared to the centralized approach, the overhead of our approach is the employment of vertical barriers. Figure 9 shows the average number of vertical barriers for the above network scenarios: network of size 10,000 meters $\times$ 250 meters at node density $\lambda=0.005,0.0075$, and 0.01 . The number of vertical barriers increases linearly with the width of each vertical strip. But we do not need to make the strip width too large and employ a large number of vertical barriers. Based on the observations for Figure 8, the barrier improvement ratio quickly levels off after the width of each vertical strip reaches a certain point. This provides a guideline to choose the width of the vertical strips. The proper width for a given network scenario can be obtained by simulation before the application of our algorithm. For example, the barrier improvement ratio saturates after the width of the vertical strips reaches 150 meters, at which point the number of vertical barriers is less than 10 for all three densities.

\section{CONCLUSION}

We study the strong barrier coverage of a randomly-deployed sensor network on a long irregular strip region. Previous research has only shown the critical conditions of weak barrier coverage (intruders take congruent paths) and how to construct sensor barriers to detect intruders crossing the strip within a small bounded region. We want to ensure that intruders cannot cross the strip undetected no matter how they choose their crossing paths. To this end, we make two main contributions that provide theoretical foundations and practical algorithm for the construction of strong barriers in a sensor network. Specifically, we obtain the critical conditions for strong barrier coverage in a strip sensor network, filling the gap in the understanding of the critical conditions for barrier coverage. Our results show that there is a critical width-to-length ratio (width being the logarithm of the length) beyond which strong barriers start to emerge in the strip. Below the critical width to length ratio, there is no strong barrier coverage, i.e., there is a crossing path along which an intruder can cross the network undetected. Based on this result, we further devise an efficient distributed algorithm to construct disjoint barriers in a sensor network with low delay, communication overhead, and computation cost. The simulation results show that our distributed algorithm can significantly improve the strength of the local barrier coverage in each segment over global barrier coverage.

\section{ACKNOWLEDGMENTS}

We thank the anonymous reviewers for their helpful comments. Benyuan Liu was partly supported by the National Science Foundation under grant CNS-0721626. Jie Wang was partly supported by the National Science Foundation under grants CCF-0429906 and CNS-0709001.

\section{REFERENCES}

[1] A. Chen, S. Kumar, and T.-H. Lai. Designing localized algorithms for barrier coverage. In Proceedings of ACM Mobicom, 2007.

[2] T. H. Cormen, C. E. Leiserson, R. L. Rivest, and C. Stein. Introduction to Algorithms (Second Edition). MIT Press and McGraw-Hill, 2001.

[3] M. Franceschetti, O. Dousse, D. Tse, and P. Thiran. Closing the gap in the capacity of random wireless networks. In Proc. of Information Theory Symposium (ISIT), 2004.

[4] D. Gage. Command control for many-robot systems. In Proc. of the Nineteenth Annual AUVS Technical Symposium (AUVS-92), 1992.

[5] G. R. Grimmett. Percolation. Springer, 1999.

[6] S. Kumar, T. H. Lai, and A. Arora. Barrier coverage with wireless sensors. In Proc. ACM Mobicom, 2005.

[7] X.-Y. Li, P.-J. Wan, and O. Frieder. Coverage in wireless ad-hoc sensor networks. IEEE Transactions on Computers, 52(6):753-763, June 2003.

[8] B. Liu and D. Towsley. A study on the coverage of large-scale sensor networks. In The 1st IEEE International Conference on Mobile Ad-hoc and Sensor Systems, 2004. 
[9] S. Meguerdichian, F. Koushanfar, M. Potkonjak, and M. B. Srivastava. Coverage problems in wireless ad-hoc sensor networks. In Proc. IEEE Infocom, pages 1380-1387, 2001.

[10] S. Meguerdichian, F. Koushanfar, G. Qu, and M. Potkonjak. Exposure in wireless ad-hoc sensor networks. In ACM Mobile Computing and Networking, pages 139-150, 2001.
[11] A. Schrijver. Combinatorial Optimization. Springer, 2003.

[12] G. Veltri, Q. Huang, G. Qu, and M. Potkonjak. Minimal and maximal exposure path algorithms for wireless embedded sensor networks. In Proc. of $A C M$ Sensys, 2003. 